\title{
Hacia una caracterización del ciberlenguaje adolescente: el caso de la multimodalidad en Facebook
}

\section{Towards a characterization of adolescents' cyberlanguage: the case of multimodality on Facebook}

PABLO SCOPONI

Kimpel Hall 425, Fayetteville, AR 72701

phscopon@email.uark.edu

Orcid: https://orcid.org/0000-0001-6939-5336

Recibido: 15/11/2019. Aceptado: 5/12/2019

Cómo citar: Scoponi, Pablo (2019), "Hacia una caracterización del ciberlenguaje adolescente: el caso de la multimodalidad en Facebook”, Revista Estudios del

Discurso Digital (REDD), 2: 101-128.

DOI: https://doi.org/10.24197/redd.2.2019.101-128

Resumen: El artículo da cuenta de una investigación doctoral en marcha que estudia las interacciones digitales de adolescentes argentinos en la red social Facebook. El estudio explora la producción lingüístico-discursiva de usuarios de tres ciudades de la República Argentina: Buenos Aires, Córdoba y San Carlos de Bariloche. En relación con la construcción de su identidad "en línea" dentro del grupo de pares, nos hemos focalizado en la multimodalidad presente en la comunicación digital de estos adolescentes, en particular, la relación entre foto y texto. Con tal fin, se analiza un total de 1560 posteos realizados en los muros de los participantes durante abril de 2012. El corpus contempla un total de 91 adolescentes ( 45 hombres y 46 mujeres) que aceptaron participar del estudio, el cual se centró en posteos que ya habían sido emitidos al momento de la toma de datos (i. e.: diciembre de 2015). El análisis (cualitativo y cuantitativo) ha dado resultados parciales que, por un lado, muestran diferencias significativas entre las publicaciones de hombres y mujeres, así como en cuanto al lugar de emisión y, por el otro lado, recoge también similitudes en cuanto cómo y para qué se comunican los adolescentes de las tres ciudades consideradas.

Palabras clave: ciberlenguaje; adolescentes; Facebook; CMC; multimodalidad; identidad.

\footnotetext{
Agradezco a mi directora de tesis doctoral Mabel Giammatteo quien me ha guiado en el desarrollo de esta investigación. Al mismo tiempo, reconozco como propios los errores que pudiera contener este artículo.
} 
Abstract: This article addresses an ongoing doctoral research that delves into the online interaction of Argentinean adolescents on Facebook. The study explores users' linguistic-discursive production from three distinctive regions of Argentina: Buenos Aires, Córdoba and San Carlos de Bariloche. In relation to the construction of their online identity inside the peer group, we have focused on the multimodality present in these adolescents' digital communication, especially the bond between pictures and text. The analysis studies 1560 messages posted on their Facebook wall during April 2012. The corpus comprises posts produced by 91 adolescents ( 45 males and 46 females). Facebook users had already posted those messages when they were asked (and accepted) to take part in the study (i. e., December 2015). The analysis (qualitative and quantitative) displays partial results, which are significant regarding gender and location, and show evidence of similar behavior regarding the way and purpose of their communication.

Keywords: cyberlanguage; adolescents; Facebook; CMC; multimodality; identity.

Sumario: Introducción. Algunas consideraciones acerca de la red social Facebook. Comunicación en la red social Facebook. De los mensajes instantáneos al muro de Facebook. La red social Facebook como "locus plástico" de la interacción digital adolescente. El posteo multimodal: mediador de la identidad adolescente. Marco metodológico. Descripción de la muestra. Discusión. Resultados. Conclusiones.

Summary: Introduction. Some facts about Facebook social network. Communication on Facebook social network. From instant messages to the Facebook wall. Facebook social network as a "plastic locus" of adolescent digital interaction. Multimodal posting: mediator of adolescent identity. Methods. Data description. Discussion. Results. Conclusions.

\section{INTRODUCCIÓN}

En las plataformas Web 2.0, la Comunicación mediada por ordenador (CMO) se presenta principalmente en forma multimodal (Herring, 2019; Kress y Van Leeuwen, 2017/2001). Un claro ejemplo lo conforma Facebook, la red social más grande en el mundo (Statista.com, 2019). En ella, los usuarios intercambian posteos bien de manera semipública a través del muro que ofrece la red social, o bien de forma privada a través de su chat.

Considerados los mayores iniciadores de los cambios lingüísticos (Eckert, 2005: 94), los adolescentes en Facebook co-construyen significado mediante su actividad comunicativa. En principio, hemos partido inicialmente de la noción de Goffman (2001/1959: 27) sobre “actuación” (del inglés, "performance”) en una conversación cara a cara: actividad total de un participante, en una ocasión determinada, que sirve para influir de algún modo sobre los otros participantes. En términos generales, en relación con la presentación de la persona en la vida cotidiana, Goffman (2001/1959) postula que la interacción cara a cara se presenta como una representación escénica que persigue realizar 
actuaciones para que los participantes se influencien entre sí. Partiendo de estas consideraciones sobre la comunicación "offline", nos abocaremos a la idea de una "actuación digital" de los adolescentes argentinos en la red social Facebook. Esta red da cuenta de esa actividad comunicativa al albergar en su muro rastros de sus interacciones, por ejemplo, a través de "texto escrito oralizado" (Yus, 2010: 197) y suplementado con elementos gráficos, visuales, auditivos, o audiovisuales (Herring, 2019: 25).

En este trabajo exploratorio, que a la vez es parte de un estudio más amplio sobre el lenguaje adolescente de la República Argentina, atenderemos a la relevancia de ciertos aspectos de las prácticas digitales diarias que contribuyen a la conformación de su identidad dentro del grupo de pares. Por otro lado, esta investigación se enmarca en la propuesta de Herring recientemente reformulada (2019: 26) sobre el Análisis del discurso mediado por ordenador (ADMO). Al respecto, hemos tenido en cuenta una serie de herramientas metodológicas que se esgrimen en torno a cuatro niveles lingüísticos: estructura, significado, gestión de la interacción y comportamiento social.

En particular, en este artículo se explorarán dos cuestiones esenciales de la comunicación adolescente: a. cómo se comunican y b. para qué se comunican. En los apartados siguientes, desarrollaremos aspectos teóricos relativos a la red social Facebook, a la interacción multimodal y a la identidad adolescente. Luego de contextualizar nuestro objeto de estudio, presentaremos la metodología adoptada seguido del análisis cualitativo de los datos que componen nuestro corpus, así como los datos cuantitativos más relevantes. Por último, ilustraremos los resultados del estudio en función de las variables seleccionadas y discutiremos tanto sus límites como potenciales desarrollos futuros.

\section{Algunas CONSIDERACIONES ACERCA DE LA RED SOCIAL FACEBOOK}

Boyd y Ellison (2007) postulan que todas las redes sociales comparten características que les permiten a sus usuarios: construir un perfil público o semipúblico dentro de un mismo sistema; articular una lista de otros usuarios con quienes están conectados; ver y recorrer tanto su propia lista de contactos como aquellos establecidos por otros dentro de ese sistema.

En particular, partiendo de esas características básicas, Facebook además de ser una red social, en la actualidad es la red social con mayor 
éxito en el cibermundo (Statista.com, 2019) y ha sido empleada por diferentes grupos desde sus orígenes (Tagg y Seargeant, 2016). En un principio, sus actores eran los estudiantes universitarios para quienes había sido creada la plataforma. Años más tarde, el uso de Facebook presenciaría un viraje hacia todas las franjas etarias y hacia personas de actividades muy diversas. No obstante, en este artículo, nuestro interés estará centrado en la actividad discursiva de la franja etaria adolescente, individuos que se encuentran en el comienzo del año lectivo 2012.

En cuanto al aspecto vincular, existen tres maneras de tener visibilidad en Facebook: a través de un "perfil", de un "grupo" o de una "página”. El área principal de trabajo de Facebook (Ureaña et al., 2011) está conformada por el perfil de cada usuario. Desde allí, se puede configurar la información que se desea mostrar u ocultar, según el grado de privacidad deseado. Este esquema de interacción con otros usuarios ha atraído a millones de adeptos.

Los usuarios (personas físicas) deciden a quiénes aceptan como amigos en su "cuenta personal” de Facebook. Simultáneamente, existen "páginas" que, por lo general, están orientadas a otro público, como a las empresas o a la creación y venta de productos. Aunque los perfiles están limitados por un número de amigos a quienes se puede aceptar para que pasen a formar parte de una lista, las "páginas" carecen de esa restricción. En cuanto a los "grupos", se pueden generar tres clases: "abiertos” al público; "cerrados” a solamente un grupo específico de contactos; y "secretos", en los que es necesario conocer el grupo para formar parte de él.

Por último, en cuanto al diseño de la interfaz de Facebook, Vela Delfa (2017) describe cuatro funcionalidades que ofrece la red social a sus usuarios. Entre ellas, destacaremos, por un lado, la generación de contenidos y, por el otro, su intercambio en el muro de los usuarios. Asimismo, explica que los perfiles se actualizan de forma regular en un flujo de información multimodal: texto, foto, video y audio.

Cabe destacar que nuestra investigación aborda solamente los posteos que los adolescentes realizan en su muro y su característica multimodal. No hemos considerado ni los grupos ni las páginas para este estudio. 


\section{COMUNICACIÓN EN LA RED SOCIAL FACEBOOK}

La red social Facebook está atravesada por un orden jerárquico que estructura la actividad discursiva y práctica semiótica de sus usuarios. Esta organización les permite postear y compartir mensajes compuestos de textos escritos, imágenes, videos o de audios. De esta manera, teniendo en cuenta los aspectos vinculares entre amigos y un carácter lúdico y local de los grupos a los que pertenecen, la arquitectura de Facebook (Piscitelli et al., 2010) fomenta la publicación personal de cada usuario dentro de cada grupo de pares.

Nada atrae más a un usuario que saber que sus amigos están compartiendo tiempo juntos utilizando una misma red social (Tagg y Seargeant, 2016). ¿Tal vez, por ello, la red social semi-pública Facebook ha atraído a tantos usuarios? ¿Resulta curioso que Facebook atraiga a usuarios cuya lista de contactos "online” coincide en mayor medida con la lista de amigos "offline”?

En este orden de ideas, Tagg y Seargeant (2016) agregan que los usuarios deben utilizar un conjunto de recursos para de alguna manera lograr presentarse ante terceros y así generar vínculos interpersonales que sean duraderos; y concluyen en que existe una relación entre el usuario y la manera en que percibe a su destinatario invisible e imaginado, quien se corporiza a través de la práctica semiótica y gracias a los recursos del contexto que ofrece Facebook.

\section{De los Mensajes instantáneos al Muro de FACEBooK}

La comunicación o mensajería instantánea (del inglés “instant messaging”) retoma la idea original del telégrafo en cuanto a su inmediatez. Esta característica prevalece en las funciones que ofrecen los sistemas actuales de mensajería, cuyas propiedades han sido enriquecidas con otro tipo de funciones. Dado que la Internet se convirtió en un innegable espacio para la interacción social (Zhao, 2005), una vez que se instaló en la vida de los ciberusuarios, el correo electrónico y los comunicados peer-to-peer (antes utilizados para compartir información entre académicos) serían superados poco después para transitar al siguiente paso en la evolución: una era de comunicación permanente. La comunicación instantánea hoy se caracteriza por su inmediatez, pero también por una serie de elementos distintivos que la posicionan como 
instrumento clave mediador entre los participantes: a) interconectividad, b) cero costo y c) facilidad.

Previo a la Internet, , los mensajes de texto (enviados a través de un teléfono) entre adolescentes podían ser establecidos entre un emisor y un único destinatario. Luego se logró perfeccionar ese mecanismo para que un único emisor enviara un mismo mensaje simultáneamente a más de un destinatario. Ello propició la comunicación en tiempo real entre grupos de adolescentes a la vez que favoreció su interconectividad. Adicionalmente, las aplicaciones actuales le permiten al emisor corroborar si el mensaje fue recibido o si fue recibido y leído por el destinatario.

El cibermundo ha generado gradualmente nuevos aportes a la vida de los individuos. En particular, uno de ellos comprende el intercambio de información, en sus inicios, a través del Sistema de Tiempo Compartido Compatible del Instituto de Tecnología de Massachusetts (Hooley et al., 2012: 7) hasta llegar, en la actualidad, a la emisión de los parlamentos característicos de la red social Facebook, WhatsApp o Twitter. Es evidente que la comunicación instantánea es uno de los aportes que no solo ha coexistido en el mundo virtual, sino que también ha evolucionado junto con la aparición de las distintas redes sociales. Por ejemplo, en solamente unas décadas, los intercambios de textos breves escritos fueron aumentando el contenido de multimodalidad (Cantamutto, 2017: 93). En cuanto al mensaje emitido, el ciberusuario prioriza el contenido del mensaje y velocidad de emisión, por lo que decide dejar de lado la forma y otros aspectos estructurales de su comunicación. Por ejemplo: “ns vms to2 n 1 rato” o "hst prnt!".

\section{1. Evolución de la norma}

Entre las características distintivas de la redacción adolescente presentes en la mensajería instantánea, se destacan (Giammatteo y Albano, 2009) el uso de abreviaciones; la elisión de vocales y consonantes no significativas; la ausencia de puntuación final, salvo signos de exclamación o interrogación de cierre; la utilización de la minúscula para abrir los parlamentos; la combinación de caracteres alfanuméricos; entre otros. En general, la necesidad de inmediatez desplaza el interés por la forma del mensaje. Es decir, el usuario le da mayor importancia a transmitir el contenido en menor tiempo sin importarle cuánto “altere” la norma lingüística. 
Crystal (2008) apunta que, en ese tipo de interacciones en las que el individuo juvenil textea un mensaje a su destinatario, se pone de manifiesto un rasgo lúdico y que los jóvenes rápidamente se dan cuenta de que una de las actividades más divertidas es jugar con la lengua, es decir, con su estructura gramatical, fónica e incluso gráfica y léxicosemántica. Este uso cibernético se sustenta en la simplificación del lenguaje y, aunque a simple vista podría pensarse en una "deformación" de la expresión escrita, desde una mirada ciberpragmática, esta escritura oralizada (Yus, 2010) es considerada una de las características distintivas de la comunicación instantánea. Dicha tendencia no solo enmarca las prácticas discursivas adolescentes, sino que se ha convertido en un elemento identitario de sus intercambios cibernéticos.

\section{LA RED SOCIAL FACEBOOK COMO “LOCUS PLÁSTICO” DE LA INTERACCIÓN DIGITAL ADOLESCENTE}

Facebook constituye un espacio que responde a las necesidades de sus usuarios: se comprime o se expande de acuerdo al uso que se le dé. Por ello, aquí lo consideraremos un "locus plástico", que emula a la "plasticidad neuronal", entendida como la capacidad del cerebro de reaccionar y de adaptarse a los estímulos ambientales (Núñez Herrejón et al., 2015/2010). De la misma manera que los individuos manifiestan esta plasticidad, Facebook parece hacer lo propio, adaptándose a sus miembros según sus necesidades.

Dada la relevancia que tiene para sus usuarios expresar sus emociones, Facebook ha expandido, por ejemplo, su tradicional "Me Gusta” a cinco opciones. Es decir, en este locus plástico no hay solo modificaciones a nivel de la estructura, sino también a nivel del modo de expresión de sus usuarios. Ellos también han modificado la forma canónica de redacción para comunicar sus visiones con mayor rapidez.

Aunque el cambio de paradigma respecto de las adaptaciones gráficas al idioma español pueda traer ciertos resquemores puristas, hoy día resultaría extraño pensar en adolescentes sin acceso a estos medios de sociabilización o sin la libertad con la que redactan sus textos que, en parte, reflejan su identidad.

Al estudiar a los adolescentes en MySpace — previo a la aparición de Facebook-, Boyd (2007) notó dos rasgos recurrentes en las entrevistas que realizó: 
When I ask teenagers why they joined MySpace, the answer is simple: "Cuz that's where my friends are." Their explanation of what they do on the site is much more vague: "I don't know... I just hang out." Beneath these vague explanations is a clear message: the popularity of MySpace is deeply rooted in how the site supports sociality amongst preexisting friend groups. Teens join MySpace to maintain connections with their friends.

Sobre la base de esa observación, Boyd (2007) concluye que los adolescentes se unen a las redes sociales, primero, porque sus amigos están ahí y, segundo, porque disfrutan de pasar el rato en ese entorno. Boyd plantea que las redes sociales se construyen y mantienen a través de la participación de sus miembros y descarta la mera observación como una actividad que atraiga más adeptos. Al respecto, distingue cuatro propiedades que sostienen la trama textual de las redes sociales, a saber: persistencia (las conversaciones pueden seguirse asincrónicamente), buscabilidad (la identidad mediada textualmente puede buscarse), replicabilidad (el texto puede copiarse y pegarse en otro sitio) y audiencias invisibles (no se puede precisar quién exactamente accede a nuestro texto, ni tampoco cuándo ni dónde).

Siguiendo este orden de ideas, sumado a estas características que Boyd enuncia, podemos apreciar otro factor común a las interacciones de Facebook: un locus que alberga dichas prácticas sociales y que se nutre multimodalmente. Por ejemplo, allí se intercambian mensajes de texto con cierta característica de la comunicación cara a cara, a los cuales Crystal (2008) llama "textspeak", dado que el texto utilizado contiene ciertos cambios morfológicos que pretenden semejar el lenguaje oral. Esta práctica socio-virtual sería parte fundante de dicha interacción juvenil, la cual en cierta medida ayudará a delinear la construcción de la identidad adolescente.

\section{EL POSTEO MULTIMODAL: MEDIADOR DE LA IDENTIDAD ADOLESCENTE}

El análisis de la CMT es un fenómeno complejo. Sin embargo, partiendo de la noción de que todas las interacciones son multimodales (Norris, 2004: 1), al analizar el discurso digital de Facebook, nos encontramos con un panorama muchísimo más simple. De esta manera, al pensar a la CMT como otra forma de interacción humana, también 
reconocemos a la comunicación en las redes sociales como multimodal (Herring, 2019; Kress y Van Leeuwen, 2017/2001).

A diferencia de la interacción cara a cara, en Facebook solamente se cuenta con dos de todos los modos semióticos presentes en la vida "offline”. En particular, nos referimos al visual y al auditivo (Paulwels, 2012), dado que por ahora (año 2019) esta red social aún carece de algún dispositivo que transmita o (re)genere aromas, texturas o sabores. La relación diádica que se establece entre quien postea y su potencial audiencia pareciera ir tejiéndose en una red de posteos multimodales que contienen al acto comunicativo.

Por tanto, en esta interdependencia de lo posteado y lo visto/escuchado, las redes sociales se esgrimen como el contexto escénico que ofrece un respaldo tanto técnico como social de múltiples formas de expresión y de co-construcción de la identidad (Rueda-Ortiz y Giraldo, 2016: 43). En este respecto, en sentido amplio, las interfaces digitales, y en sentido estricto, el muro de Facebook, se constituyen como espacios propicios para quienes activamente deseen presentarse ante una audiencia determinada; y para quienes puedan expresarse utilizando los recursos expresivo-simbólicos disponibles en este entorno determinado (Rueda-Ortiz y Giraldo, 2016). Es decir, cómo los individuos buscan ejercer control sobre los demás a través de la manera que eligen para presentarse y así generar y controlar las impresiones en la percepción de sus amigos.

Esta necesidad humana de querer controlar la percepción del "self" en la audiencia se verá exacerbada por una característica inherente al adolescente: la necesidad de mantener cierta distancia respecto de la relación con sus padres (Garrett y Williams, 2005: 40) para acercarse a un grupo de pa(d)res que idealmente le devolverá la auto-imagen de quien necesitará ser para mantener su membresía dentro del grupo. Al respecto, Krauskopf (2010) enuncia que

La identidad social podemos entenderla como el producto de la interacción de distintos componentes identitarios que se articulan para insertarse en determinado espacio sociopersonal y dan sustento a la expresión de comportamientos y relaciones.

Esa devolución grupal que reciben continuamente parece fortalecer su identificación y contribuir a su autoestima, mediante la asociación con 
los pares, el sentirse reconocidos, valorados, queridos y, por ende, aceptados.

Al respecto del hábitat de la red social Facebook, la validación del mundo emocional del adolescente, se manifiesta de forma textual, pero también multimodal (e. g., a través de texto y de imagen). Un mensaje puede ser emitido, por ejemplo, tanto con la palabra escrita cuanto con una foto o con un video, o mediante la selección y configuración de varios modos (Jewitt, 2016: 70).

\section{1. El posteo multimodal: consideraciones para nuestro análisis}

Sobre la base de lo expuesto, conjeturamos que para realizar un posteo, o responderlo, se puede postear texto, memes, videos, fotos, “Me Gusta" (como respuesta), etc., o mediante la combinación esos modos. Entendemos que ese comportamiento digital representa una conducta diaria adolescente en el muro de Facebook, en un intento de validar al o esperar validación del grupo de pares.

A modo de ejemplificación, se presenta el siguiente posteo (imagen con texto) seguido del impacto que causó en los demás miembros:

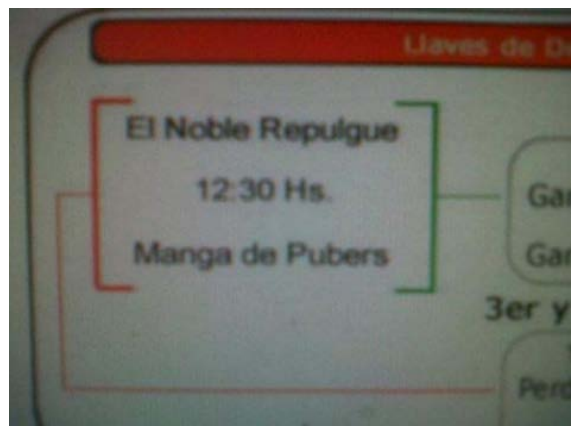




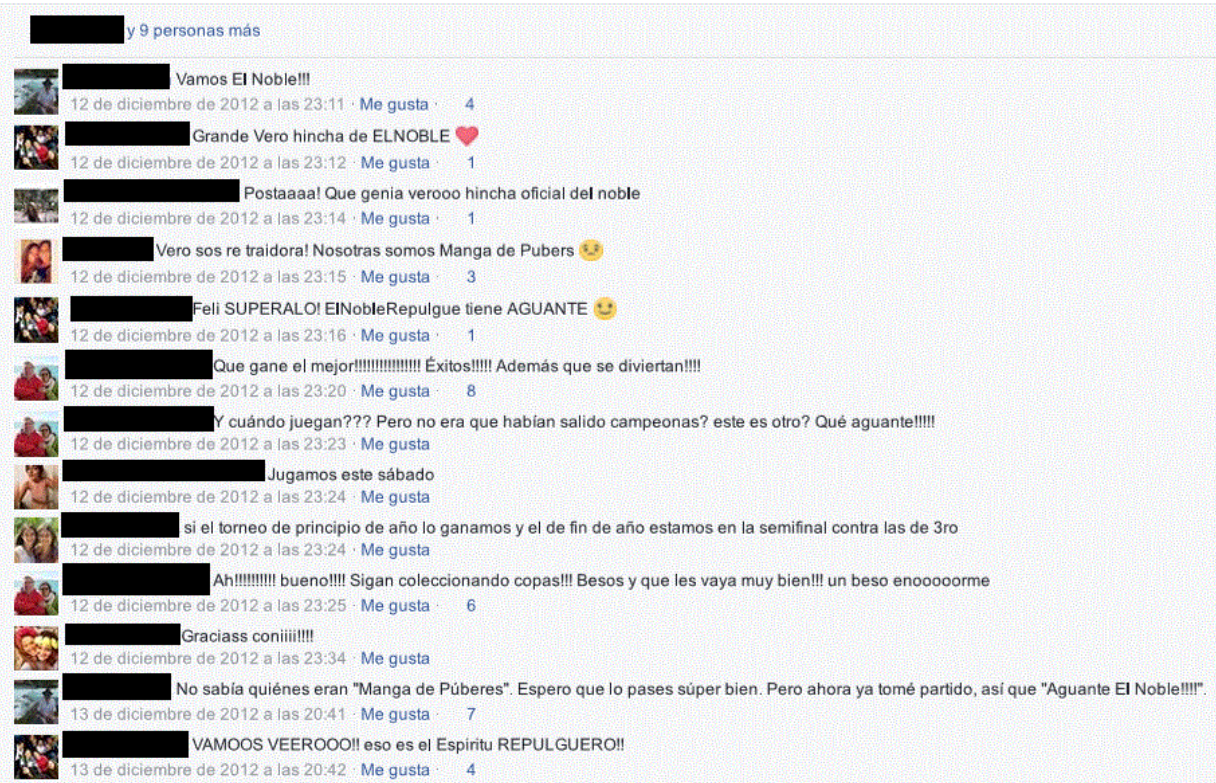

El posteo inicial remite a la imagen de una casa de empanadas (i. e., una comida argentina), llamada "El Noble Repulgue". Como resultado, el usuario de esta cuenta de Facebook recibió diversos tipos de respuesta. En primer lugar, se aprecia la gradación de Me Gusta en dos niveles, con un total de treinta y cinco reacciones:

1. un nivel primario, con un total de diez Me Gusta inmediatamente debajo de la foto posteada, y

2. un nivel secundario, con algunos Me Gusta a la derecha de casi todos los posteos (texto o texto con emoticones) de respuesta.

Seguido al nivel primario y en forma de cascada, comienzan las validaciones con texto o texto y emoticones. Un ejemplo de léxico validante lo encontramos en la exclamación "Postaaaa!”. Este lexema procedente del lunfardo (Conde, 2011), aquí utilizado como "verdaderamente” o “de verdad”, presenta mayúscula inicial y alargamiento de la última, y está cerrado por un signo de exclamación, a diferencia de la siguiente exclamación, carente de su signo de cierre.

Otra forma de validación lo conforma el uso de mayúsculas plenas para destacar el entusiasmo: “VAMOOS VEEROOO!!”. En este último posteo, también se observa el alargamiento de ciertas vocales para acentuar la fuerza ilocutiva propia de la exclamación y para emular la 
subida tonal en las silabas destacadas (no solo las tónicas, es decir, en este caso la primera de cada vocablo).

Por último, se observa el uso de tres emojis. Cada uno de ellos acompaña al texto tecleado al que suceden. En un intento de conferir mayor capacidad expresiva al enunciado, esos usuarios han decidido cerrar sus posteos con esas figuras dotadas de una capacidad expresiva que emulan a los gestos humanos.

Este comportamiento multimodal, ya sea en el posteo inicial o en la cadena remática de posteos subsiguientes, da cuenta de la interacción lingüístico-discursiva de los adolescentes de nuestro corpus y que se manifiesta a través del muro de la red social Facebook. Asimismo, se observan las respuestas validantes de los amigos de ese usuario particular. Sin importar si están de acuerdo o no con el texto de la imagen inicial, los adolescentes responden a temáticas que los atañen a ellos como grupo (i. e., "Manga de Pubers"). Esto es, se unen para ir hacia un mismo objetivo, en este caso, expresar su acuerdo o desacuerdo sobre esa etiqueta, y para hacerlo utilizan varios modos (i. e., imagen y texto tecleado).

Al respecto, cabe aclarar que los posteos de nuestro corpus son solamente los que inicializan la cadena multimodal. Es decir, no hemos tomado en cuenta las respuestas de los amigos de cada adolescente consultado, sino la forma en la cual cada uno se presenta al comienzo de dicha cadena. Por último, esta investigación no pretende realizar un análisis pormenorizado del texto tecleado, sino centrarse en la interacción entre texto y los demás modos presentes en nuestro corpus (en particular, el caso de las fotos posteadas en el muro de Facebook), en función de la validación de cada adolescente dentro del grupo de pares de cada una de las regiones que conforman este estudio.

\section{MARCO METODOLÓGICO}

Como se ha planteado en los apartados anteriores, esta investigación se enmarca en el estudio del lenguaje digital y tiene en cuenta el contexto de la acción comunicativa, y en particular, el análisis del discurso mediado por computadora. Con la intención de caracterizar al lenguaje adolescente argentino, hemos analizado las prácticas socio-digitales (mes de abril de 2012) de 91 adolescentes en sus respectivos muros de la red social Facebook. El estudio de esta construcción y co-construcción social del lenguaje adolescente, se centra, desde un enfoque probabilístico, de 
las ocurrencias del corpus, en los aspectos multimodales (en particular, la relación entre foto y texto), así como también los pragmático-discursivos.

Para el análisis metodológico, hemos partido en principio de las propuestas sobre la investigación sociolingüística y pragmática de SilvaCorvalán (2017: 39):

El paradigma metodológico que se identifica más fácilmente con estudios sociolingüísticos es aquel cuyo objetivo es la descripción y explicación de ciertos usos lingüísticos variables característicos de una comunidad.

En segundo término, según Fielding, Lee y Blank (2017/2008), una de las maneras de abordar el análisis de las redes sociales es mediante la detección del contenido temático ("TCA": “Thematic Content Analysis”). Es decir, cualquier análisis de contenido en el cual las variables remiten a la frecuencia de ocurrencia de temas específicos (Popping, 2017: 331).

Por último, en cuanto a las variables multimodales, hemos tomado el desarrollo de Jewitt (2016: 70) sobre multimodalidad. En general, las características esenciales de una comunicación multimodal, y en particular, los rasgos que deben analizarse al momento de abordar el análisis de la imagen (Jewitt, 2016: 80). Según dichas consideraciones, y dadas las particularidades del muro de Facebook, en nuestro análisis hemos tenido en cuenta (a) el impacto del empleo de un ensamble de modos en la construcción de significado; (b) el foco sobre una actividad manifestada dentro de un entorno social compartido por los usuarios de esta red; y (c) la relevancia, por un lado, en la selección de ciertos modos que atiendan a generar significado y, por el otro, en la interacción que se establece entre un modo y otro, como parte del mismo tejido multimodal. En este respecto, hemos hecho especial foco en el uso de fotos con texto y sin él, como se verá más adelante.

En este orden de ideas, y siguiendo a Silva-Corvalán, en nuestra descripción del habla digital adolescente, nos hemos centrado en un uso consensuado de los modos de hablar de los tres grupos de pares recolectados de tres ciudades de la República Argentina: Buenos Aires, Córdoba y San Carlos de Bariloche. Por ello, nuestro análisis se orienta hacia las variables con mayor frecuencia de ocurrencia, las cuales darían cuenta de aquellas marcas identitarias compartidas por los miembros de los tres grupos de pares, dado que adoptar una conducta comunicativa 
que se aleje de los modos de habla compartidos por grupo, implica cierto rechazo hacia los miembros de esa comunidad. En particular, como apunta Silva-Corvalán (2017: 24):

Abandonar los modos de hablar del grupo al que se pertenece implica sentimientos de rechazo hacia ese grupo y pérdida de parte de la identidad.

De acuerdo a lo planteado por Silva-Corvalán (2017: 39) sobre los pasos que se suelen seguir para un análisis sociolingüístico de esta índole, presentaremos las preguntas de investigación, el objetivo general y los objetivos específicos. Posteriormente, detallaremos la recolección de la muestra y los aspectos generales del corpus. Por último, expondremos una descripción detallada de la muestra con sus correspondientes ejemplos.

\section{1. Preguntas de investigación}

Este estudio intenta responder a las siguientes preguntas de investigación:

1. ¿Cuáles son los temas más relevantes que aúnan a cada una de estas poblaciones en términos de su producción multimodal?

2. ¿Qué tipo de producción multimodal exhiben estos jóvenes y cuáles son las características principales de sus posteos?

3. ¿Para qué se comunican los adolescentes oriundos de tres provincias de la República Argentina: Buenos Aires, Córdoba y Río Negro en la red social Facebook?

\section{2. Objetivos}

Teniendo en cuenta nuestras preguntas de investigación, atenderemos a los siguientes objetivos:

1. Identificar ejes temáticos recurrentes en los posteos relevados.

2. Describir cualitativa y cuantitativamente los hallazgos sobre la producción multimodal adolescente en función de las variables “género” y “diatópica” para explorar si existen diferencias significativas.

3. Identificar la intención comunicativa de la actividad multimodal adolescente. 


\section{3. Recolección de la muestra y el aspecto ético}

Para conformar la muestra, a principios de 2015 se convocó a los potenciales padres o familiares de los “adolescentes 0”. Se les explicó de qué se trataba el estudio sobre el análisis del habla digital de adolescentes argentinos en Facebook y se les pidió que les preguntaran a los jóvenes de la familia si estaban dispuestos a formar parte de este estudio. También se les aclaro que el investigador solamente accedería al año 2012 del muro de cada adolescente, para copiar los posteos y pegarlos en una planilla Excel para su posterior análisis. A todos los padres o familiares se les aclaró que el adolescente debería enviarle al investigador una solicitud de amistad vía Facebook. Solo tres adultos de los 51 consultados en persona o telefónicamente respondieron a la invitación. Algunos hicieron preguntas sobre cómo era la investigación, pero luego no volvieron sobre el tema. Otros aseguraron que le pedirían a sus hijos o sobrinos que contactaran al investigador a través de Facebook, pero el investigador no recibió ningún pedido de amistad. Los demás no respondieron a la propuesta.

Una vez que los tres adultos contactaron al investigador con los adolescentes, el investigador le envió a cada uno el consentimiento informado para que (vía chat privado de Facebook) aceptaran formar parte del estudio. Cada uno de los familiares (uno por provincia) establecieron el contacto inicial con los tres "adolescentes 0". Los tres familiares eran conocidos del investigador, pero este no conocía a los “adolescentes 0", todos de género femenino. Luego de haber accedido a participar en la investigación, las tres adolescentes viralizaron la propuesta a sus compañeros de colegio.

De esta forma, se obtuvo un total de tres grupos de pares, uno por provincia, con participantes masculinos y femeninos cada uno. La edad de los participantes rondaba entre 16 y 17 años en 2012. Al sumar la cantidad de participantes que aceptaron el consentimiento informado, se logró un total de 91 informantes, los cuales ya habían cumplido 18 años cuando accedieron a participar en el estudio. Es importante resaltar que la muestra recolectada es previa al pedido de colaboración, para evitar que los adolescentes se sintieran observados y que por ello adaptaran o modificaran su forma de comunicarse a una diferente de la cotidiana.

Por último, seleccionamos a un informante de cada provincia para hacer un conteo inicial de todos los posteos realizados durante el año 2012. Luego de verificar que el mes de abril de los tres informantes 
contenía casi el doble de la cantidad de posteos respecto del resto de los meses, decidimos seleccionar ese mes como representativo de la muestra.

\section{4. Procesamiento de la muestra}

En primer término, luego de ingresar los posteos en una planilla de Excel (paquete Microsoft), se agregaron las variables correspondientes a la producción de posteos con texto propio y texto importado de la Internet, los aspectos pragmático-discursivos, así como los aspectos multimodales, para realizar los conteos de las ocurrencias de cada uno de ellos, respectivamente. Seguido a dicho etiquetado cualitativo, se procedió a calcular las frecuencias, teniendo en cuenta el total de posteos.

En segundo término, en cuanto a las variables sociodemográficas, solamente se han tomado en cuenta las diferencias socio-educativas y geográficas como significativas, dado que el corpus, por un lado, está formado por adolescentes que pertenecen a un mismo grupo etario, y por el otro, presenta un número similar de participantes tanto masculinos (45) como femeninos (46).

En último término, se utilizó el mismo programa para determinar la direccionalidad y magnitud del comportamiento de los datos. En dicho análisis, se exploraron las variables que conforman el análisis. Asimismo, se empleó ese programa para graficar los resultados.

\section{DESCRIPCIÓN DE LA MUESTRA}

Con esta muestra, se intenta caracterizar el habla adolescente desde un enfoque sociolingüístico, lingüístico propiamente dicho y pragmáticodiscursivo, haciendo énfasis en la interacción entre texto y los demás modos que registra nuestro corpus, para atender a los usos multimodales que los adolescentes presentan en el muro de Facebook. En sentido amplio, el corpus pertenece a la comunidad lingüística del habla argentina, Sin embargo, en sentido estricto, el corpus está compuesto por tres subcorpora que reúnen muestras de tres variedades dialectales argentinas (i. e., el español bonaerense, cordobés y patagónico).

El corpus de cada región posee no solo particularidades geográficas distintas, sino también presenta características sociolectales diferentes. Así, por ejemplo, tanto el subcorpus de la provincia de Córdoba como el de la provincia de Río Negro corresponden a hablantes de la clase social 
media, mientras que el subcorpus de la provincia de Buenos Aires, a los de la clase alta.

Con la intención de proporcionar una mirada que aporte al campo de investigación del lenguaje digital adolescente en las redes sociales, hemos seleccionado una serie de índices (i. e., de humor, de ubicación y el de emociones y sentimientos) que permiten comprender con mayor precisión las manifestaciones de los adolescentes. Cabe mencionar que los resultados que arrojan los conteos correspondientes nos permiten únicamente corroborar la frecuencia de aparición, pero no así determinar ningún tipo de causalidad sobre las variables que se ponderan en esta investigación, para lo cual se debería recurrir a un estudio de corte experimental.

\section{DisCUSIóN}

\section{1. El posteo multimodal: epígrafe de la imagen}

En este estudio, denominamos “epígrafe de la imagen” al texto que precede a una imagen (e. g., foto) posteada para abrir una cadena de posteos, ya sea que aparezcan seguidos de tales posteos o que hayan quedado carentes de respuesta. A continuación, mostraremos dos ejemplos:
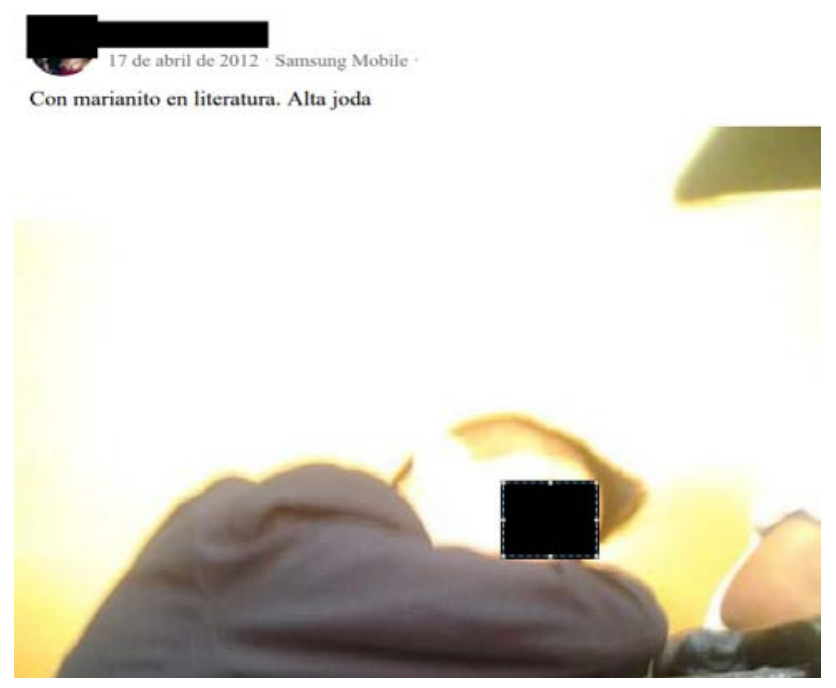
La adolescente que posteo esta imagen de su amigo durmiendo en la clase de Literatura se incluye automáticamente en la foto a través del epígrafe de esta imagen, al enunciar "Con marianito en literatura" en ese epígrafe. Es decir, asumimos que es ella quien saca la foto desde su "Samsung mobile", dato que aparece a la derecha de la fecha en la parte superior de la imagen. Cabe aclarar que además esta foto aparece en su muro de Facebook. Luego, la segunda oración, "Alta joda”, le da al posteo multimodal un giro de 180 grados. Si la foto no hubiera formado parte del posteo, la interpretación del mensaje habría sido uno completamente alejado del rasgo ironía que se intenta proyectar, o al menos, hubiera sido un algo ambiguo.

Del mismo modo, en la siguiente foto la adolescente expresa su cariño o amor hacia sus amigas, tal vez, de forma un tanto hiperbólica, dado que no hay una gama de grises, sino que rotundamente "las ama”. Refuerza esa idea con el alargamiento de vocales y consonantes, seguido de un emoticón. De esa manera, el vocablo "perraaaaaaasss" queda envuelto entre dos elementos con una polaridad opuesta. En este juego retorico de hipérboles y redundancias, "perraaaaaaasss" se resignifica automáticamente y, dada la proxemia respecto de los demás elementos, termina cargándose con la misma polaridad.
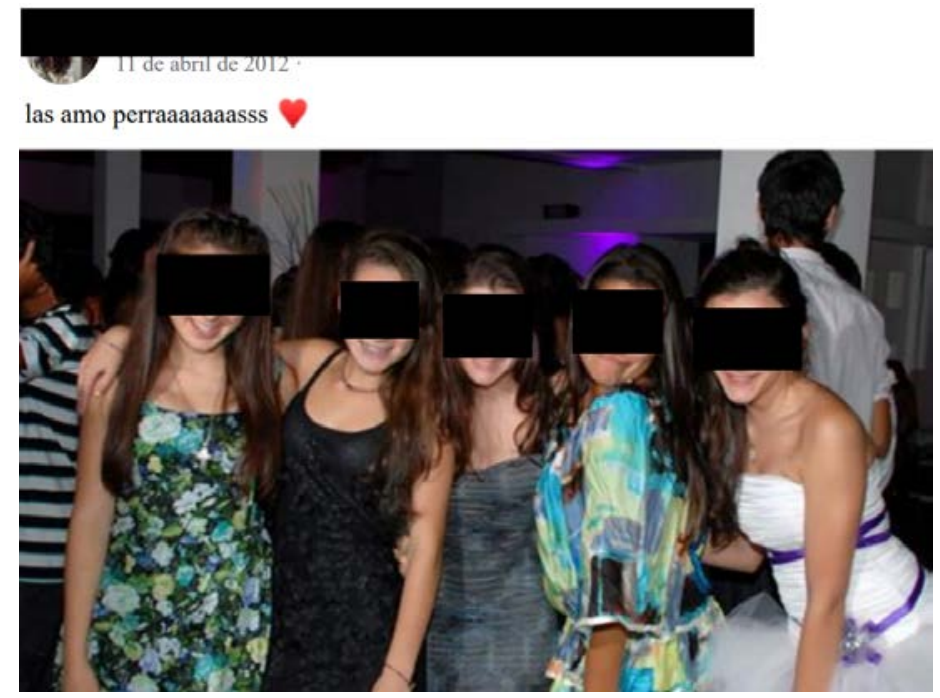
Nuestro corpus presenta tanto fotos con epígrafes como fotos sin él. Sin embargo, hemos relevado más fotos con epígrafe. Cuando está presente, el epígrafe parecería redireccionar la interpretación del universo de inferencias que podrían establecerse sin el soporte textual. Además, cuando están presentes, remiten a los rasgos “compañía”, "sentimiento” o "estados de ánimo". Es decir, el epígrafe completa el sentido de la imagen compartida. Sin embargo, argumentamos que no agrega, sino que complementa. Parecerían complementarse mutuamente, foto y texto, respecto del contenido proposicional que cada uno de estos dos elementos denota por separado. Por ello, consideramos que el epígrafe de la imagen es parte inherente en este par semiótico, y que solo cuando ambos elementos comparten un mismo posteo, aportan a la connotación de una realidad que se actualiza para proyectarse en su audiencia de la manera esperada.

\section{2. Multimodalidad inversa: Emojización de la comunicación adolescente}

Como hemos mencionado en apartados anteriores, la comunicación adolescente en la red social Facebook se desarrolla en un medio plástico. Juntos, los usuarios y Facebook afrontan un aprendizaje diario. Por ejemplo, en un principio, la plataforma solo contaba con el botón "Me Gusta” para expresar aprobación o gusto por un posteo. Luego, Facebook agregó el resto de las opciones con las que cuenta en la actualidad (e. g., el botón para expresar sorpresa). Con sus prácticas, los adolescentes podrían llegar a influir significativamente en la estructura de la red.

Al mismo tiempo que Facebook incorpora cambios en su morfología, adaptándose a las necesidades de los jóvenes, ellos también parecen hacerlo. De manera consciente o inconsciente, los adolescentes han incorporado a sus posteos de fotos (propias o de terceros) una serie de gestos, los cuales se asemejan a las caras o expresiones de los emojis o emoticones. A continuación, se presenta un ejemplo:

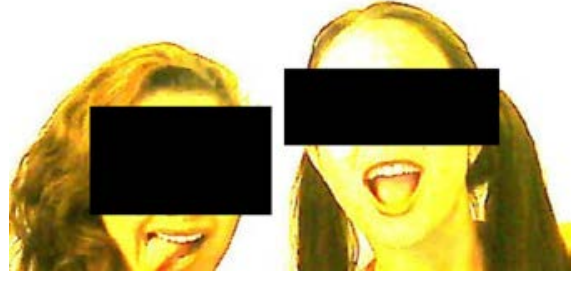


Como se aprecia en la imagen de arriba, las dos adolescentes juegan a "poner caras" que implican sacar la lengua. Veamos qué ofrece Facebook al respecto:
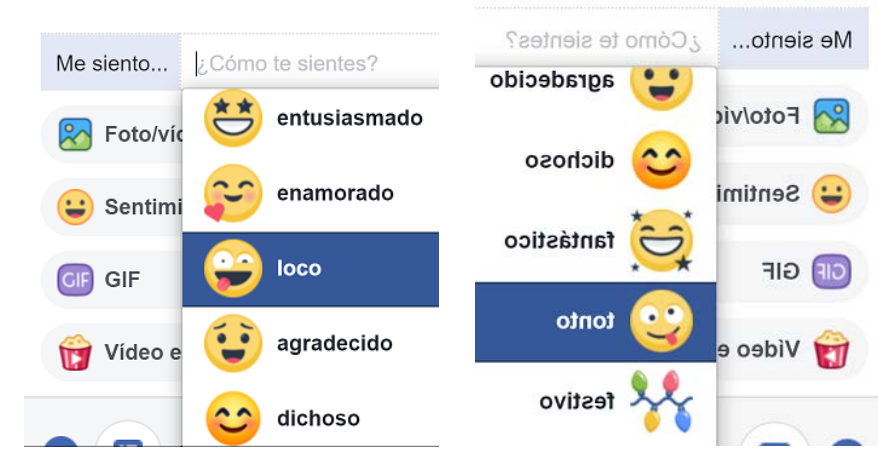

¿Estaremos frente a alguna forma de "multimodalidad inversa”? O, ¿simplemente los jóvenes se divierten "haciendo caras" en esta red social?

En nuestro estudio, la afluencia de fotos con este rasgo es radical y lo hemos denominado "emojización” o “emoticonización”, como parte de un posible proceso "multimodalización inversa”.

Aquí debajo hemos incluido otro ejemplo de este rasgo identitario de este grupo etario:



Algo que debemos destacar es que, en la mayoría de las fotos relevadas con este rasgo, se aprecia la presencia de una emojización "à deux". Esto es, los actores suelen ser dos, salvo en ocasiones en las cuales hay más participantes. Independientemente de este proceso, los adolescentes tienen a adoptar conductas parecidas, que a su vez parecen 
ir generando una red de comportamientos esperados en el contexto de interacción que ofrece el muro de la red social Facebook.

\section{Resultados}

En este apartado, discutiremos los resultados parciales que arrojó el estudio cualitativo y cuantitativo. El análisis del corpus muestra un total de 1560 posteos relevados, que fueron realizados por 91 jóvenes del grupo etario adolescente. Todos los adolescentes residían en la Argentina en 2012 y eran usuarios activos de la red social Facebook. A continuación, se presentan los resultados iniciales del corpus, según el género y el origen:

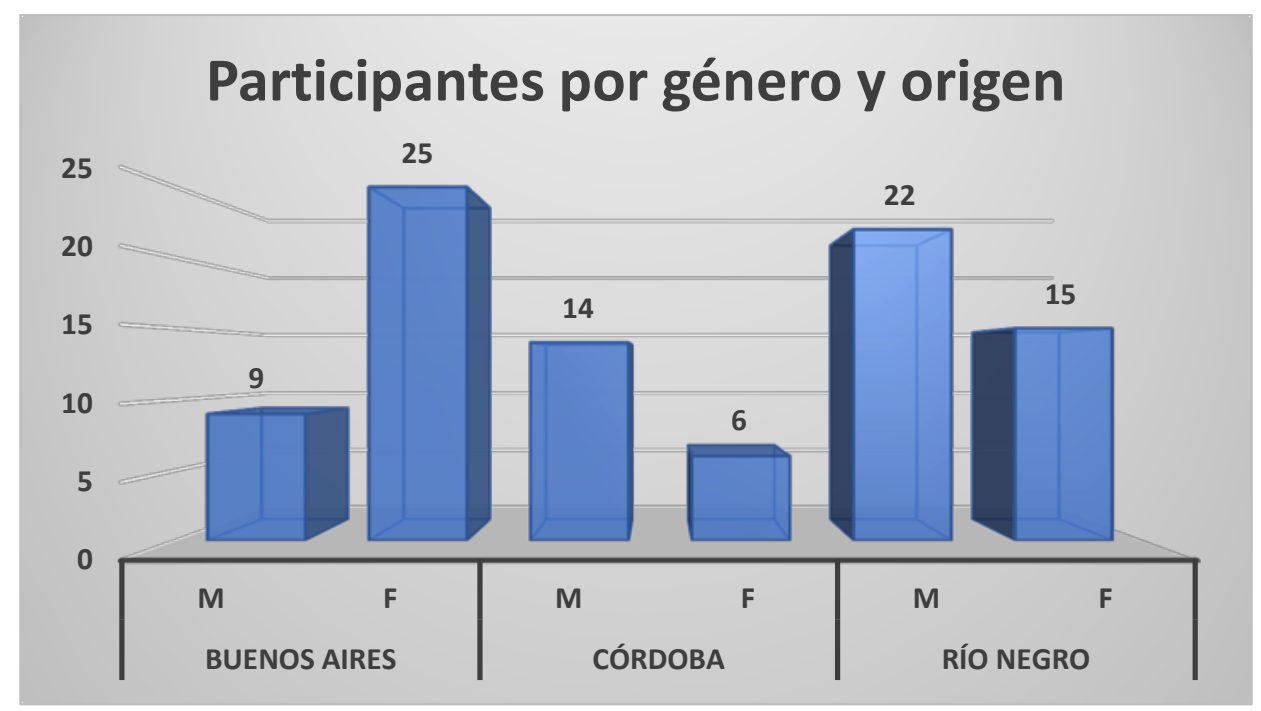

Figura 1. Participantes por género y origen

Cabe aclarar que, en un principio, los adolescentes de Buenos Aires y de Río Negro se mostraron más dispuestos a participar y formar parte en la investigación. Resultaron ser mayor en número de participantes por origen que los de Córdoba, quienes fueron los más reticentes a participar. Sin embargo, cuando realizamos el estudio de orden cuantitativo, la provincia de Córdoba fue la que obtuvo los mayores porcentajes en producción pragmático-discursiva y multimodal en general. 
Los 91 adolescentes realizaron 1560 posteos, de los cuales se desprende la siguiente distribución.

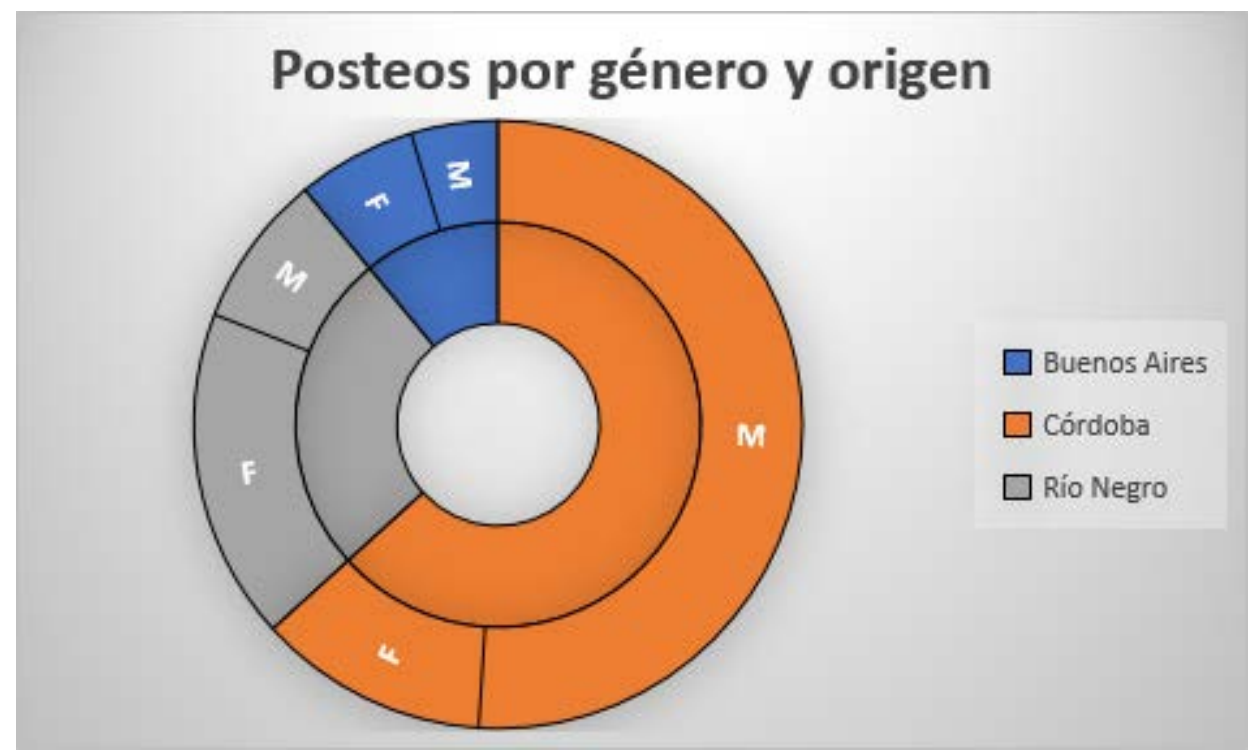

Figura 2. Posteos por género y origen

A pesar de que los adolescentes de Córdoba eran en número relativamente menor al de las otras dos provincias, superó a Buenos Aires y a Río Negro en la cantidad de posteos publicados. Obtuvo un total de 987 mensajes posteados. Es decir, Córdoba conforma el 63\% del total de los posteos. Luego le sigue Río Negro con un $26 \%$ y, en último lugar, Buenos Aires con un 11\% de posteos. Tanto en Río Negro (68\%) como en Buenos Aires (58\%), las mujeres fueron las que más postearon en ese mes. Sin embargo, por un $81 \%$ en Córdoba los hombres superaron a las mujeres.

En cuanto a los 1560 posteos, el 65\% fue producido mediante texto tecleado por los propios adolescentes, mientras que el 35\% restante remite a texto o enunciados "importados" o "apropiados". De esa manera hemos considerado llamar a todos los posteos que se importaron desde algún sector de la Internet.

Respecto de cómo se comunican los adolescentes, los datos revelan que los jóvenes producen tanto posteos con texto como posteos sin texto. En cuanto a los primeros, las variables que se tuvieron en cuenta para 
analizar cada posteo fueron las siguientes: enunciados importados (34\%), emoticones (15\%), fotos (18\%), memes (5\%) y videos (4\%). En cuanto a la variable "audio", no se registra ningún posteo relacionado. En cuanto a texto propio que inicializan los posteos, se registran los siguientes datos:

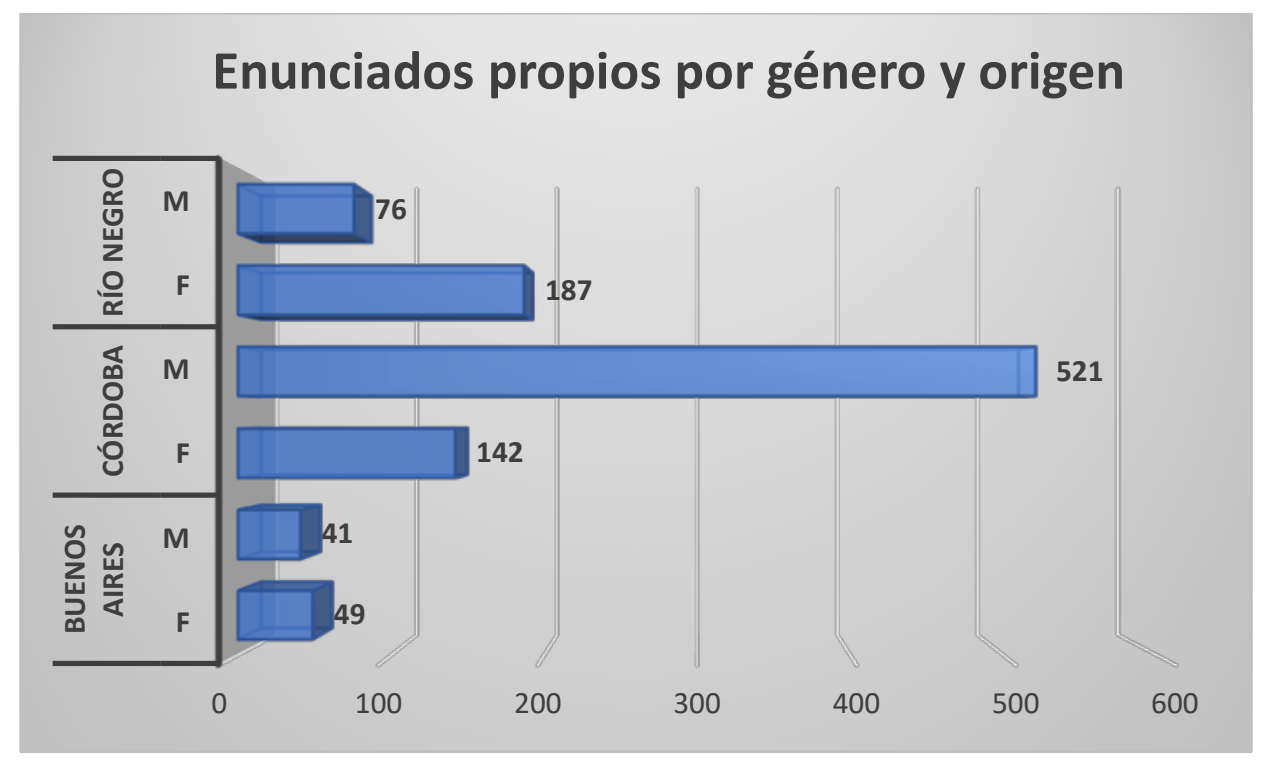

Figura 3. Enunciados propios por género y origen

Como se aprecia, los hombres de Córdoba han producido más posteos con texto propio tecleado que el resto de los participantes. El total de este tipo de posteos es 1016, es decir, que los adolescentes prefieren (65\%) generar texto propio que utilizar texto proveniente de otras fuentes. En cuanto al 35\% restante, está compuesto por material tomado de la Internet. Entre esos ítems, se destacan las letras de canciones, imágenes (memes y fotos), frases célebres, y enlaces de Internet a videos.

Respecto de los posteos multimodales, las fotos propias representan el 65\% de la actividad digital adolescente. Cabe mencionar que no se han tenido en cuenta las fotos que amigos postean en un muro ajeno o las publicaciones que incluyen el Álbum. A continuación, nos detendremos aquí para abordar los resultados, a saber: 


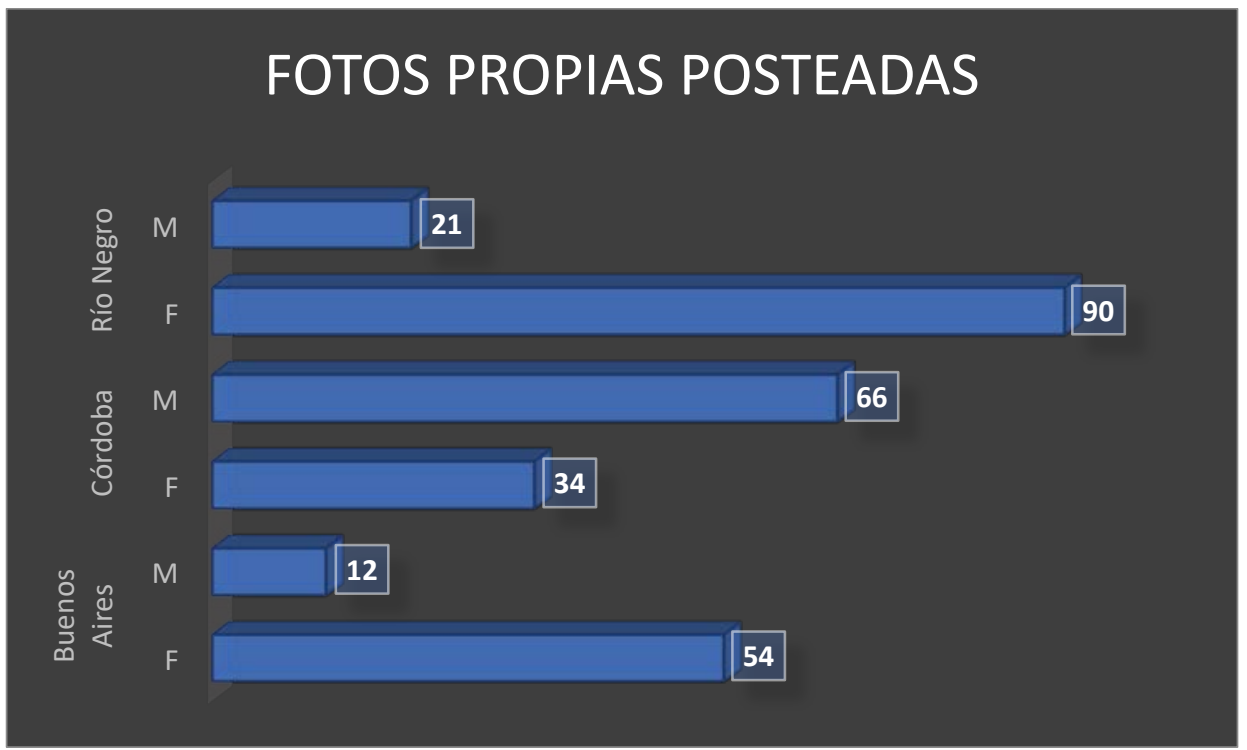

Figura 4. Fotos propias posteadas

En cuanto a las fotos, tuvimos en cuenta si estaba precedida por epígrafe o no. El estudio indicó que los adolescentes postearon un 62\% de fotos con epígrafe y un 38\% de fotos sin epígrafe. Respecto de las primeras, tanto las mujeres de Río Negro (32\%) como los hombres de Córdoba (23\%) representan a los adolescentes con mayor actividad en ese aspecto. Sin embargo, en general, las mujeres registran un $69 \%$ de fotos con epígrafe respecto de los hombres con solo un 31\% de actividad. Asumimos que las mujeres prefieren resaltar una parte de la foto o agregan información que no está presente en ella.

Por último, en cuanto a para qué se comunican, los datos arrojan en primer término que buscan expresar sentimientos o emociones. Un total de 1017 de los 1560 posteos fueron etiquetados con esa variable. En segundo término, postean para generar humor (436), y en tercer término, para compartir su ubicación en tiempo real (305) con los demás amigos de su grupo de pares. Respecto de estas tres variables, los hombres de la provincia de Córdoba son quienes registran el mayor porcentaje. 


\section{Conclusiones}

Este estudio ofrece una mirada sobre cómo los adolescentes argentinos se comunican en el muro de la red social Facebook. Los adolescentes de las tres provincias analizadas utilizan Facebook como un espacio naturalmente multimodal que viabiliza su proceso de socialización, a la vez que les permite estar en contacto con los demás miembros del grupo de pares.

El análisis ha arrojado luz sobre algunos aspectos relativos al comportamiento adolescente en esta red social. En términos generales, los hallazgos dan cuenta de la preferencia por utilizar más el texto tecleado por ellos mismos que aquel tomado de alguna parte de la Internet (e. g., enlaces a sitios web, letras de canciones, etc.). En términos particulares, los adolescentes oriundos de la ciudad de Córdoba exhiben un mayor porcentaje de actividad en el muro de Facebook, a pesar de ser el grupo de pares con menos cantidad de participantes del estudio.

Otro dato significativo lo representa la publicación de imágenes. En particular, respecto de las fotos que postean los adolescentes, se registra un creciente despliegue de conductas “emojizantes”. Hemos conjeturado que es posible que la exposición diaria y continua a la CMO (o CMT) haya de alguna manera influido sobre sus prácticas comunicativas en su intento de promover la construcción de significado y en consecuencia generado cierto grado de multimodalidad inversa, en la cual los adolescentes parecen modelar las expresiones faciales de los emoticones o emojis, originalmente creadas para modelar las humanas.

En cuanto a las limitaciones de este artículo, no se pondera aquí un análisis que incluya una investigación de corte correlacional o que ofrezca miradas basadas en regresiones, dada la fase en la que se encuentra el estudio mayor en donde está inscripto el presente estudio. Por ello, solo nos hemos abocado al modo visual en función de los dos tipos de texto relevados en nuestro corpus: el propio y el importado (en su carácter de epígrafe).

Este estudio podría resultar útil para trazar nuevas líneas de investigación que pretendan abordar el análisis de la multimodalidad en las redes sociales y busquen profundizar de modo correlacional las variables "texto" e "imagen", en función de las variables "expresar sentimientos o emociones”, "generar humor” y "compartir ubicación en tiempo real”. Asimismo, podrían explorarse las características constitutivas de la imagen en función de las tres variables anteriores. Es 
decir, cuando el rasgo [+ emoji] está presente en la foto, ahondar en la utilización del espacio donde se desarrolla la acción (dentro de una instalación o al aire libre); el máximo de actores intervinientes (e. g., verificar si hay preferencia por un número en particular); planos más utilizados (e. g., primer plano o plano americano); entre otras tantas propuestas.

\section{BIBLIOGRAFIA}

Boyd, D. (2007). "Why youth (heart) social network sites: the role of networked publics in teenage social life". En MacArthur Foundation Series on Digital Learning Youth, Identity, and Digital Media Volume (ed. David Buckingham). Cambridge, MA: MIT Press.

Boyd, D. y Ellison, N. (2007). "Social network sites: definition, history, and scholarship". En Journal of Computer-Mediated Communication, 13(1), Indiana: Indiana University. http://jcmc.indiana.edu/vol13/issue1/boyd.ellison.html (15-1119).

Cantamutto, L. (2017). “Economía, claridad y expresividad lingüísticas”. En Mabel Giammatteo, Patricia Gubitosi y Alejandro Parini (comp.), El Español en la Red Madrid: Iberoamericana, pp. 93-119.

Conde, O. (2011). Lunfardo: un estudio sobre el habla popular de los argentinos. Buenos Aires: Taurus.

Crystal, D. (2008). “Txting: frNd or foe?”. The Linguistic, 47(6), 8-11.

Eckert, P. (2005). "Stylistic practice and the adolescent social order". En Angie Willims y Crispin Thurlow (comp.), Talking Adolescence Nueva York: Peter Lang, pp. 95110.

Garcés-Conejos, P. y Bou-Franch, P. (2019). "Introduction to analyzing digital discourse: new insights and future directions”. En Pilar Garcés-Conejos y Patricia Bou-Franch (comp.), Introduction to Analyzing Digital Discourse: New Insights and Future Directions Cham: Palgrave Macmillan, pp. 3-22.

Garrett, P. y Williams, A. (2005). “Adults' perception of communication with young people”. En Willams, A. y Thurlow, C., Talking Adolescence. Perspectives on communication in the teenage years Londres: Peter Lang Publishing Inc., pp. 3552.

Giammatteo, M. y Albano, H. (2009). "El español en Internet: una mirada a su evolución en los fotologs”. En Revista Digital Universitaria, 10(3),

http://www.revista.unam.mx/vol.10/num3/art15/int15.htm (15-11-19). 
Goffman, E. (2001). La presentación de la persona en la vida cotidiana, 1a. edición, 3a. reimpresión, Buenos Aires: Amorrortu. (Obra publicada originalmente en 1959).

Herring, S. (2019). “The coevolution of computer-mediated communication and computer-mediated discourse analysis”. En Pilar Garcés-Conejos y Patricia BouFranch (comp.), Introduction to Analyzing Digital Discourse: New Insights and Future Directions Cham: Palgrave Macmillan, pp. 25-67.

Hooley, T., Marriott, J., y Wellens, J. (2012). “A brief history of online research methods". En What is Online Research? Using the Internet for Social Science Research ("What is?" Research Methods Series), pp. 7-24, Londres: Bloomsbury Academic. http://dx.doi.org/10.5040/9781849665544.ch-002 (15-11-19).

Jewitt, C. (2016). “Facebook and the discursive construction of the social network”. En GeorgaKopoulou, Alexandra y Spilioti, Tereza, The Routledge Handbook of Language and Digital Communication Nueva York: Routledge, pp. 69-84.

Krauskopf, D. (2010). “The contemporanean youth condition and the constitution identity”. En Ultima década, 18(33), 27-42. https://dx.doi.org/10.4067/S071822362010000200003 (15-11-19).

Kress, G. y Van Leeuwen, T. (2017). Multimodal discourse: The modes and media of contemporary communication. Londres: Arnold. (Obra publicada originalmente en 2001).

Norris, S. (2004). Analyzing multimodal interaction: a methodological framework. Nueva York: Routledge.

Núñez Herrejón, J., Ortiz Salinas, M. y Viveros Fuentes, S. (2015). APA: Diccionario conciso de psicología. Mexico D. F.: El Manual Moderno. (Obra publicada originalmente en 2010).

Pauwels, L. (2012). “A multimodal framework for analyzing websites as cultural expressions”. En Journal of Computer-Mediated Communication, 17(3), pp. 247265. https://doi.org/10.1111/j.1083-6101.2012.01572.x (15-11-19).

Piscitelli, A., Adaime, I. y Binder, I. (comp.) (2010). El proyecto Facebook y la posuniversidad. Sistemas operativos sociales y entornos abiertos de aprendizaje. Madrid: Ariel, Fundación Telefónica.

Popping, R. (2017). “Online tools for content analysis”. En Fielding, N., Lee, R., y Blank, G. (comp.), The SAGE Handbook of Online Research Methods London: SAGE, pp. 329-343. 
Tagg, C. y Seargeant, P. (2016). "Facebook and the discursive construction of the social network”. En GeorgaKopoulou, Alexandra y Spilioti, Tereza, The Routledge Handbook of Language and Digital Communication Nueva York: Routledge, pp. 339-353.

Thurlow, C., Lengel, L. y Tomic, A. (2004). Computer Mediated Communication: Social Interaction and the Internet. London: Sage.

Urueña, A., Ferrari, A., Blanco, D. y Valdecasa, E. (comp.) (2011). Las redes sociales en Internet, Madrid: Observatorio Nacional de las Telecomunicaciones y de la SI.

http://www.ontsi.red.es/ontsi/sites/default/files/redes_sociales-documento_0.pdf (15-1119).

Vela Delfa, C. (2017). "Coherencia, cohesión y estructura de la interacción en el discurso digital: un análisis de los intercambios en la red social Facebook”. En Mabel Giammatteo, Patricia Gubitosi y Alejandro Parini (comp.), El Español en la Red Madrid: Iberoamericana, pp. 255-277.

Rueda-Ortiz, R. y Giraldo, D. (2016). "Profile Image: ways of self-(re-) presentation on the Facebook social network”. En Walrave, M., Ponnet, K., Vanderhoven, E., Haers, J. y Segaert, B. (comp.), Youth 2.0: social media and adolescence

Yus, F. (2010). Ciberpragmática 2.0. Nuevos usos del lenguaje en Internet. Barcelona: Ariel Letras.

Zhao, S. (2005). “The digital self: through the looking glass of telecopresent others”. En Symbolic Interaction, 28(3), pp. 387-405. JSTOR. www.jstor.org/stable/10.1525/si.2005.28.3.387 (15-11-19). 